\title{
Correction to: Synchronization control for networked mobile robot systems based on Udwadia-Kalaba approach
}

\author{
Conghua Wang $($ Jinchen Ji • \\ Zhonghua Miao · Jin Zhou
}

Published online: 21 June 2021

(C) Springer Nature B.V. 2021

\section{Correction to: Nonlinear Dyn \\ https://doi.org/10.1007/s11071-021-06487-z}

The authors would like to point out the following updates to the published article:

1. The e-mail of J. Zhou should be jzhou@ @hu.edu.cn and the e-mail of J. Ji should be jin.ji@uts.edu.au.
2. In the second paragraph of Section 4.4, Eq. (21) should be Eq. (38) and equations (21), (21e) should be Eqs. (19), (38).

The original article has been corrected.

Publisher's Note Springer Nature remains neutral with regard to jurisdictional claims in published maps and institutional affiliations.
The online version of the original article can be found under https://doi.org/10.1007/s11071-021-06487-z.

C. Wang · J. Zhou ( $₫)$

Shanghai Institute of Applied Mathematics and Mechanics and Shanghai Key Laboratory of Mechanics in Energy Engineering, School of Mechanics and Engineering Science, Shanghai University, Shanghai 200072, China e-mail: jzhou@shu.edu.cn

C. Wang

e-mail: chwang92@sina.com

J. Ji

Faculty of Engineering and IT, University of Technology

Sydney, Sydney, NSW 2007, Australia

e-mail: jin.ji@uts.edu.au

Z. Miao

School of Mechatronic Engineering and Automation,

Shanghai University, Shanghai 200072, China

e-mail: zhhmiao@shu.edu.cn 